\title{
Status of the GroundBIRD Telescope
}

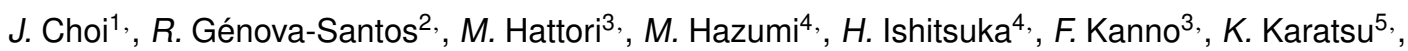

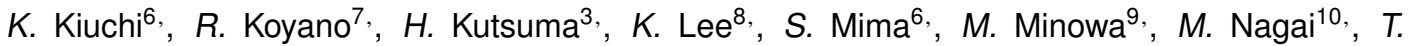

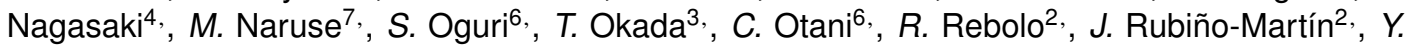
Sekimoto $^{10}$, J. Suzuki ${ }^{4}$, T. Taino ${ }^{7}$, O. Tajima ${ }^{4,11}$, N. Tomita ${ }^{9}$, T. Uchida ${ }^{4}$, and E. Won ${ }^{8, \star} M$. Yoshida ${ }^{4}$

\section{for the GroundBIRD Collaboration}

\author{
${ }^{1}$ Center for Axion and Precision Physics Research, Institute for Basic Science (IBS), Daejeon 34051 \\ ${ }^{2}$ Instituto de Astrofísica de Canarias, E38205 - La Laguna, Tenerife, Canary Islands, Spain \\ ${ }^{3}$ Tohoku University, Aramaki Aza Aoba 6-3, Aoba-ku, Sendai, 980-8578, Japan \\ ${ }^{4}$ Institute of Particle and Nuclear Studies, High Energy Accelerator Research Organization (KEK), Tsukuba, \\ Ibaraki 305-0801, Japan \\ ${ }^{5}$ TU Delft, 2600 AA Delft, Netherland \\ ${ }^{6}$ RIKEN, 2-1 Hirosawa, Wako, Saitama, 351-0198, Japan \\ ${ }^{7}$ Saitama University, Saitama-shi 338-8570, Japan' \\ ${ }^{8}$ Korea University, 145 Anam-ro, Seongbuk-gu, Seoul, 02841, Korea \\ ${ }^{9}$ The University of Tokyo, 7-3-1, Hongo, Bunkyo-ku, Tokyo 113-0033, Japan \\ ${ }^{10}$ Advanced Technology Center, National Astronomical Observatory of Japan, Tokyo 181-8588 \\ ${ }^{11}$ Department of Physics, Kyoto University, Kyoto 606-8501
}

\begin{abstract}
Our understanding of physics at very early Universe, as early as $10^{-35} \mathrm{~s}$ after the Big Bang, relies on the scenario known as the inflationary cosmology. Inflation predicts a particular polarization pattern in the cosmic microwave background, known as the $B$-mode yet the strength of such polarization pattern is extremely weak. To search for the $B$-mode of the polarization in the cosmic microwave background, we are constructing an off-axis rotating telescope to mitigate systematic effects as well as to maximize the sky coverage of the observation. We will discuss the present status of the GroundBIRD telescope.
\end{abstract}

\section{Introduction}

The present standard model of Universe [1], or smoothly expanding Universe without any non-smooth feature from the beginning, has at least two known problems. One is the observed isotropic distribution of the cosmic microwave background (CMB) temperature, down to $10 \mathrm{ppm}$ level [2] to its mean value of $2.7 \mathrm{~K}$, yet any CMB photons seen today separated by a couple of degree in the sky today were

^e-mail: eunil@hep.korea.ac.kr 
out of causal contact at the time of the last scattering before reaching to us as millimeter electromagnetic wave. This is known as the horizon problem in cosmology [3]. Also, the recent measurement on the curvature of the space of Universe indicates that Universe is very flat to sub percent level [4] and it is interpreted that the mass density in early Universe must have been fine-tuned to more than ten digits according to Friedmann equations. This is known as the flatness problem [5].

These two problems ${ }^{1}$ are considered as fine-tunning problems or initial-value problems of the standard model of Universe right after the big bang, and in order to solve them, a model of rapid, superluminal expansion of space in very early Universe, commonly called the inflationary cosmology is proposed in 1980's [6]. This exponential expansion in nature allows all photons to be in causal contact to explain the isotropic temperature of the $\mathrm{CMB}$ and flatness of the space at present. It has become clear that the ultimate test of the inflationary Universe is to search for the curl mode of the polarization in the CMB [7]. The strength of the $B$-mode polarization, that is related with the energy scale of the inflation, is unfortunately not predicted by theory precisely and commonly parameterized by the ratio, $r$ of the tensor to scalar perturbation of the power spectra of the CMB anisotropy.

The search for the primordial $B$-mode polarization of the CMB has become one of major fields in the modern cosmology and recent ground based and satellite set upper limits on $r$ to be order of 0.1 [8, 9]. Our telescope, GroundBIRD ( $B$-mode Imaging Radiation Detector) [10], is being constructed in Japan, aiming at the detection of the $B$-mode polarization down to $r$ to percent level. We will discuss advantages of our design, the construction status, and our observation plan in the following section.

\section{GroundBIRD Telescope}
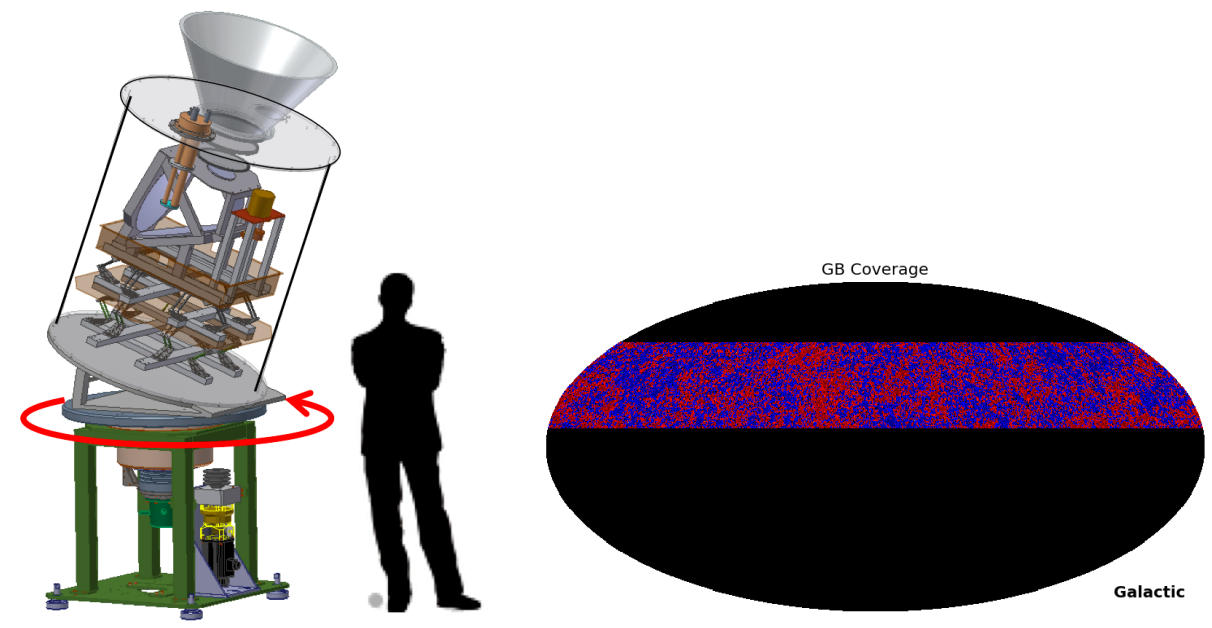

Figure 1. Left figure shows a drawing of the GroundBIRD telescope. To set the scale, a human is also drawn. Right figure show the sky coverage of the GroundBIRD telescope when it is located at the latitude of $28^{\circ} \mathrm{N}$, having a zenith angle of $20^{\circ}$.

The GroundBIRD (GB) telescope is under construction at KEK in Japan. The GB telescope adapts a fast rotation scan strategy of $20 \mathrm{rpm}$, or $120^{\circ} / \mathrm{s}$. A drawing of the GB telescope is shown at the left

\footnotetext{
${ }^{1}$ There is also a magnetic monopole problem but it assumes grand unified theories that are not settled yet and therefore not mentioned here.
} 
side in Fig. 1. As indicated here in Fig. 1, GB rotates with a finite zenith angle at the chosen site. A single rotation with a $20^{\circ}$ zenith angle forms a large toroidal coverage in the sky. On top of that, the rotation of Earth itself shifts this toroidal coverage by a constant speed of $15^{\circ} / \mathrm{h}$, making the sky coverage of the GB to be approximately $40 \%$ of the entire sky that is about 10 times larger than coverage from other ground based telescopes that use constant elevation azimuthal scan method [11]. This large sky coverage is demonstrated at the right side in Fig. 1 for the location at at the latitude of $28^{\circ} \mathrm{N}$, having the zenith angle to be $20^{\circ}$. This fast rotation scan strategy also allows to mitigate so called $1 / f$-noise effect from the baseline fluctuation of the detector. This is one of the unique features of the GB telescope. Note that rotation of the entire telescope is technically extremely challenging since at the same time, electric power lines and helium gas to the cryogenic system must be provided while the temperature of the focal plane must be stable at $250 \mathrm{mK}$. The implementation and stable operation of rotating table will be discussed in the following section.

This fast rotation enables GB to detect both the reionization bump of the multipole $\ell<10$ and the recombination peak at $\ell \approx 100$ of the $B$-mode power spectrum predicted by inflation scenario of the very early Universe. This can be demonstrated by computing the detector noise power spectrum $N_{\ell}^{B B}$ where

$$
N_{\ell}^{B B}=\left(\frac{\pi}{10800} \frac{w_{p}^{-1 / 2}}{\mu \mathrm{K} \operatorname{arcmin}}\right)^{2} \mu \mathrm{K}^{2} \text { str. }
$$

Here $w_{p}^{-1 / 2}$ is the noise in Stokes parameters $Q$ or $U$ per pixel whose solid angle $\Omega_{\text {pix }}$, gives $\sqrt{\Omega_{\text {pix }}}=1$ arcmin [12]. Using this one can estimate sensitivity and a projected GB sensitivity on the $B$-mode polarization is shown in Fig. 2. Two bottom curves (green and blue) are $B$-mode power spectra for $r=0.1$, and 0.01 . A thick (red) curve is the GB sensitivity estimation when $w_{p}^{-1 / 2}=3.5 \mu \mathrm{K}$ arcmin. A curve above (purple) is when $w_{p}^{-1 / 2}=10 \mu \mathrm{K}$ arcmin. The almost straight line (black) except right corner indicates the gravitational lensing effect, and the dashed curve is $E E$ mode of the power spectrum. Depending on the value of $r$, GB may be able to detect both bumps that are mentioned above.

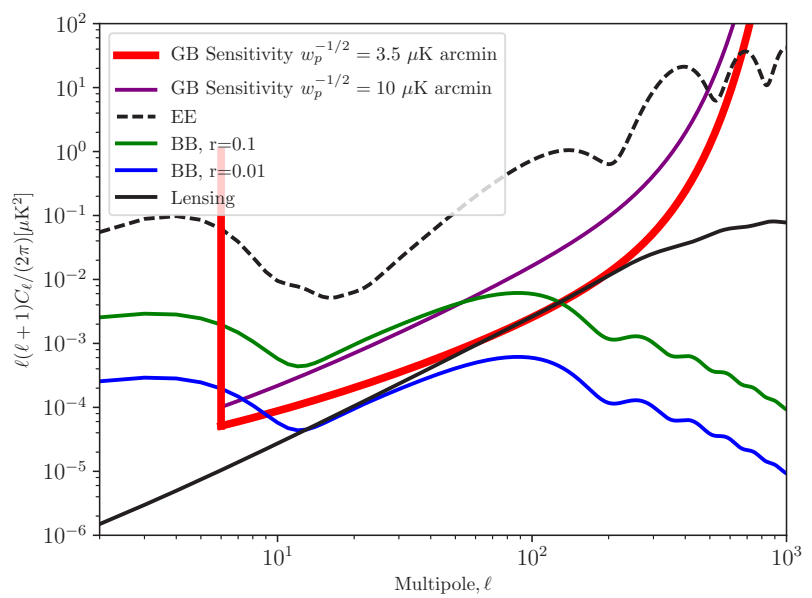

Figure 2. A sensitivity plot of the GB telescope. Two bottom curves (green and blue) are $B$-mode power spectra for $r=0.1$, and 0.01 . A thick (red) curve is the GB sensitivity estimation when $w_{p}^{-1 / 2}=3.5 \mu \mathrm{K}$ arcmin. A curve above is when $w_{p}^{-1 / 2}=10 \mu \mathrm{K}$ arcmin. The almost straight line (black) except right corner indicates the gravitational lensing effect, and the dashed curve is $E E$ mode of the power spectrum.

The optical system of GB telescope hosts a Mizuguchi-Dragone dual reflecting mirrors [13] with a focal plane. The field of view of the GB is $\pm 10^{\circ}$, and the angular resolution of each pixel is $0.5^{\circ}$ (full-width at the half maximum) for the $145 \mathrm{GHz}$ band. Including these, the design parameters of the GB telescope are summarized in Table 1. 
Table 1. List of the parameters specified in the GB telescope.

\begin{tabular}{ll} 
Aperture diameter & $300 \mathrm{~mm}$ \\
Optics & Cross Dragone, FOV $= \pm 10^{\circ}$ \\
Detector & lens-antenna-filter coupled KID \\
Frequency bands & 145 and $220 \mathrm{GHz}$ \\
Resolution & $0.5^{\circ}$ at $145 \mathrm{GHz}$ and $0.3^{\circ}$ at $220 \mathrm{GHz}$ \\
Scan speed & $120^{\circ} / \mathrm{s}$ or $20 \mathrm{rpm}$ \\
\hline
\end{tabular}

\subsection{Observation site}

The proposed observation site is located at del Teide observatory in Tenerife Island that is one of Canary Islands in Spain. The latitude of the site is $28^{\circ} \mathrm{N}$ and this allows a unique CMB observation from the Northern hemisphere. Note that most of ground-based CMB telescopes are located in South pole or at a desert in Southern hemisphere. Also, the headquarter of the Institute of Astrophysics at Canary islands (Instituto de Astrofísica de Canarias, IAC) is located near to the observation site, allowing a close collaborative local support from IAC. The proposed site is at the elevation of $2400 \mathrm{~m}$ above the sea level.

\subsection{Cryogenics}

The two rotating structure [14] consists of two sets of tables and support structure. At the bottom of the tables, rotary joints for helium gas circulation and for electric power lines are mounted. The rotary joint for helium gas circulation was specially developed but the rotary joint for electricity is a commercial one. On the table, a cryostat with cryocoolers are mounted. Other instruments such as computer, vacuum gauge, and thermometer monitor are also placed. The overall configuration can be seen in Fig. 3.

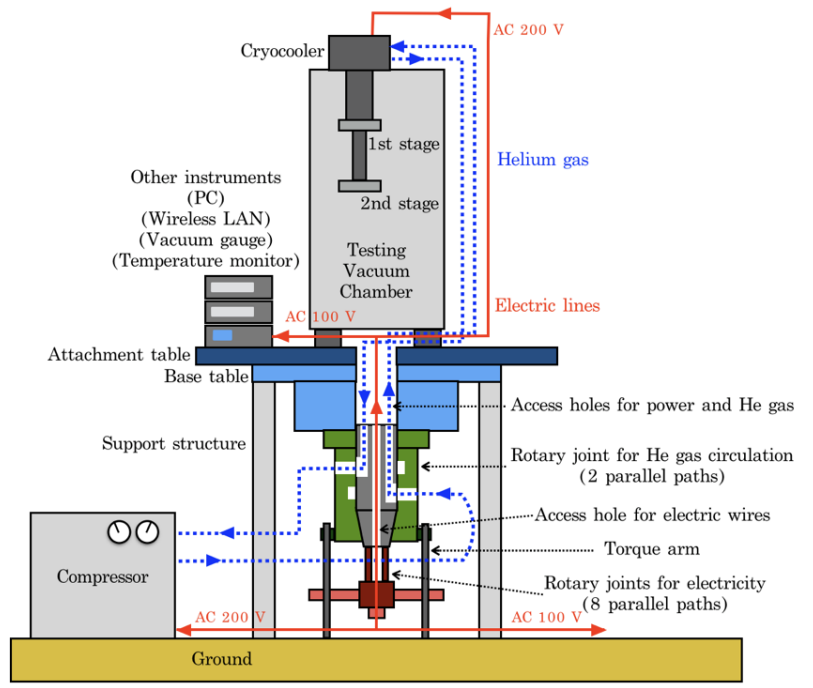

Figure 3. Layout of the system maintaining a dry cooling on a rotating table. A cryostat and other instruments are mounted on the table. A custom-made rotary joint for the helium gas transfer and a commercial rotary joint for the electricity are mounted below the table structure.

The outmost part of the cryostat is a vacuum chamber at $300 \mathrm{~K}$. Inside the vacuum chamber, 40 $\mathrm{K}$ and $4 \mathrm{~K}$ shields are nested. This allows the reflectors are at $4 \mathrm{~K}$ and that mitigates radiation noise from the mirror surface. The fake polarization through non-zero emissivity of mirror surface is also 
minimized by $1 / 100$ of the intensity compared with ambient temperature reflectors. The cryostat that allows sub-Kelvin refrigeration consists of a pulse tube cryocooler and a helium sorption cooler [15]. The cryostat on the table holds a two-stage pulse tube cryocooler from PT415-RM, Cryomech, Inc., Syracuse, NY. The cooling power for the first stage is $36 \mathrm{~W}$ at $45 \mathrm{~K}$ and for the second stage 1.35 $\mathrm{W}$ at $4.2 \mathrm{~K}$. A series of two rotary joints mentioned above maintains the simultaneous circulation of electricity and helium gas to the coolers. For the further cooling below $4 \mathrm{~K}$, a three-stage helium sorption cooler from CRC_GL10, Chase Research Cryogenics Ltd., Sheffield, UK was used at the bottom of the second stage of the cryocooler. This three stage contain sequentially ${ }^{4} \mathrm{He},{ }^{3} \mathrm{He}$, and ${ }^{3} \mathrm{He}$, and are thermally connected in series. They require cold and warm "switches" for the operation: the cold switch maintains the cryopump to adsorb the helium vapor and the warm switch raises the cryopump temperature. When the cold switch is turned on, each cold head reverts to the cold stage and when the warm switch is on, each tank is refilled with the liquid helium. A stable long-term operation at $234 \mathrm{mK}$ on the rotating table of $20 \mathrm{rpm}$ is achieved and the detailed operation is described in Ref. [16].

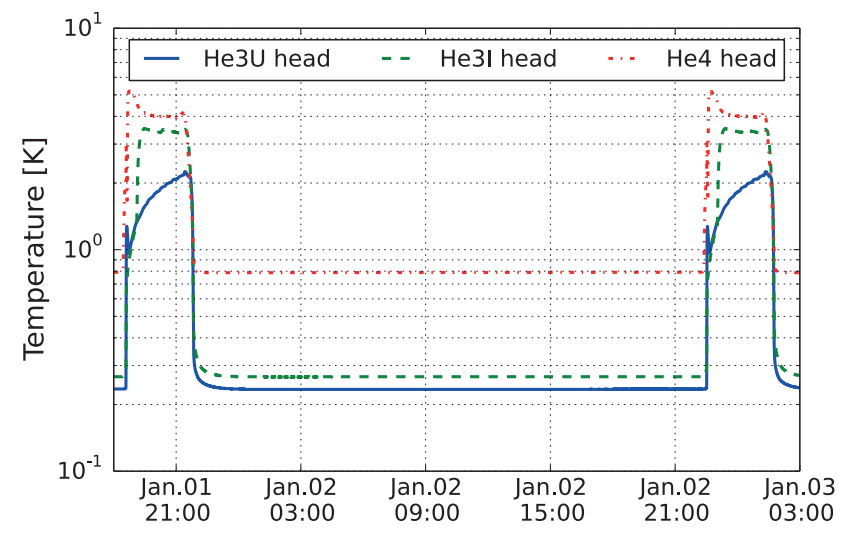

Figure 4. A refrigeration cycle for the sorption cooler on the rotation table at $20 \mathrm{rpm}$. The variation of temperature at each cold stage as a function of time is shown in this plot. Structure seen at two ends indicate the periods of warm switch that is described in the text.

The cryostat described above allows two mirrors in the Cross Dragone optics system to be at less than $4 \mathrm{~K}$ and the focal plane to be at $250 \mathrm{mK}$ and a demonstration of such capability while the cryostat is under rotation of $20 \mathrm{rpm}$ is shown in Fig. 4. The coldest head, labeled as He3U head reaches down to $234 \mathrm{mK}$ when the cryostat is under rotation for long period. This is a clear demonstration of a stable operation of the cryostat under the $20 \mathrm{rpm}$ rotation [16].

\subsection{Calibration Strategy}

Sparsely arranged straight wires are placed in front of the field of view of the telescope. A combination of these wires and the rotation of the telescope enables us to have an ideal modulation of the polarized calibration signals. This calibration can by construction be continuous during the observation period. In this way, spurious polarization can be as small as less than $0.3 \%$ level [10].

\subsection{Detector}

For the CMB millimeter photon detection, a superconducting thin film array of LC circuits, or kinetic inductance detectors (KIDs) [18] are chosen for the GB telescope. For two millimeter wave bands of GB observation, parameters of KIDs sensors are listed in Table 2. The incoming CMB radiation 

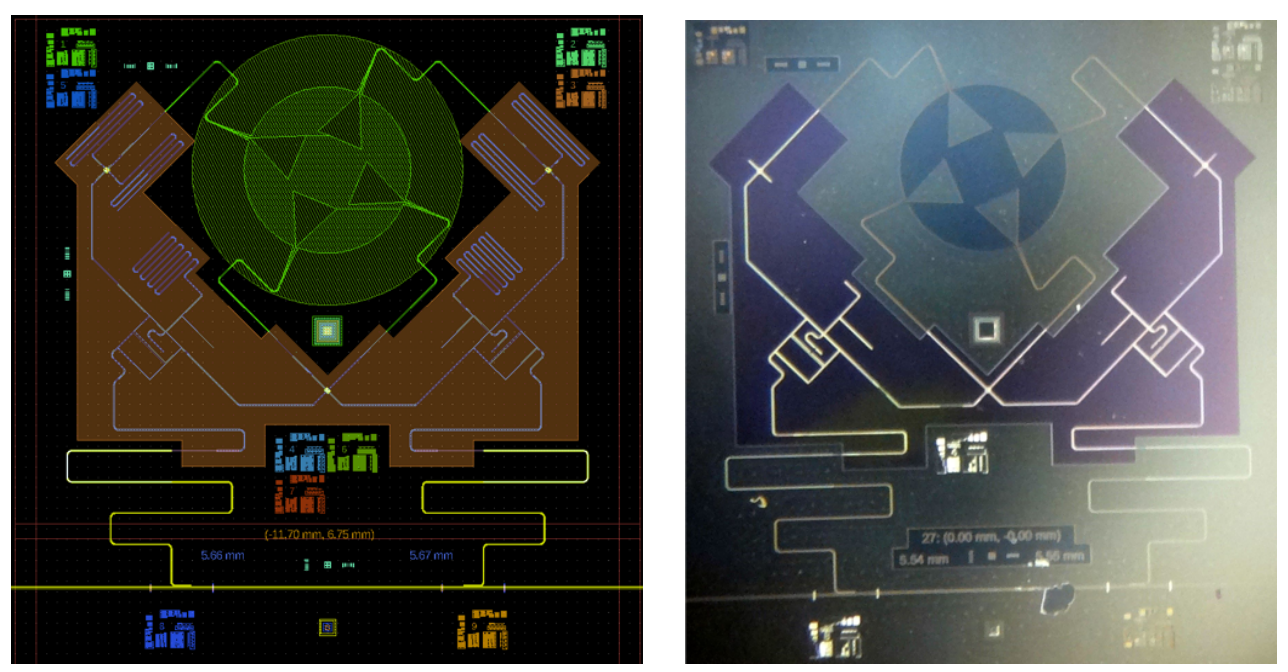

Figure 5. Our KID system mask design for simulation and fabrication (left) and a photograph [17] of the fabricated KID system using the hybrid fabrication process (right). The four triangular arms at the top-center are orthomode transducer based polarization antennas. From there, two $180^{\circ}$ hybrid are seen at the left and right sides. The two snake-like structure correspond to two KIDs and the signals from them are read out by a feedline located at the bottom that runs horizontally.

Table 2. List of the parameters specified on KIDs for the GB telescope. Note that the noise temperature in this case is integrated over the time with the photon noise limit from sky temperature.

\begin{tabular}{ccccccc}
\hline $\begin{array}{c}\text { Band } \\
(\mathrm{GHz})\end{array}$ & $\begin{array}{c}D_{\text {pixel }}(3 \lambda) \\
(\mathrm{mm})\end{array}$ & $\begin{array}{c}N E T \\
(\mu \mathrm{K} \sqrt{\mathrm{s}})\end{array}$ & $\begin{array}{c}\text { Pixel \# } \\
\text { per wafer }\end{array}$ & Wafer \# & KID \# & $\begin{array}{c}N E T_{\text {array }} \\
(\mu \mathrm{K} \sqrt{\mathrm{s}})\end{array}$ \\
\hline 145 & 6.3 & 310 & 55 & 6 & 660 & 12 \\
220 & 4.1 & 530 & 112 & 1 & 224 & 35 \\
\hline
\end{tabular}

is guided to the detector system through homemade corrugated horn. Then the polarization of CMB is detected with dual polarization antennas that is a pair of two planar orthomode transducers. Then $180^{\circ}$ hybrid selects the signal mode of TE11 and suppress TM01 mode. Since the hybrid has to be of micro strip line while antennas are coplanar ones, we design a Chebyshev transformer to match impedance. After the hybrid, a reverse process has to be made since the KID has coplanar waveguide structure, and for that another Chebyshev transformer is designed. The end-to-end simulation of the entire detector system from antenna to KID front is carried out. The transmittance for our signal TE11 is $\sim 90 \%$ for GB observation band and the crosstalk of TE1 $1 \leftrightarrow$ TE1 1 and TM01 $\rightarrow$ TE1 1 are found to be negligible.

The actual fabrication of the KID sensor system is a complicated process due to the fact that it contains both coplanar and micro strip line. A hybrid fabrication approach is applied for the fabrication: KID and feedline are fabricated at RIKEN and millimeter-wave circuits are fabricated at National Astronomical Observatory of Japan. A prototype KIDs with and without antenna circuit using this hybrid fabrication approach are made and showed similar performance between with and without antenna circuits. This demonstrates the validity of the hybrid fabrication approach. 

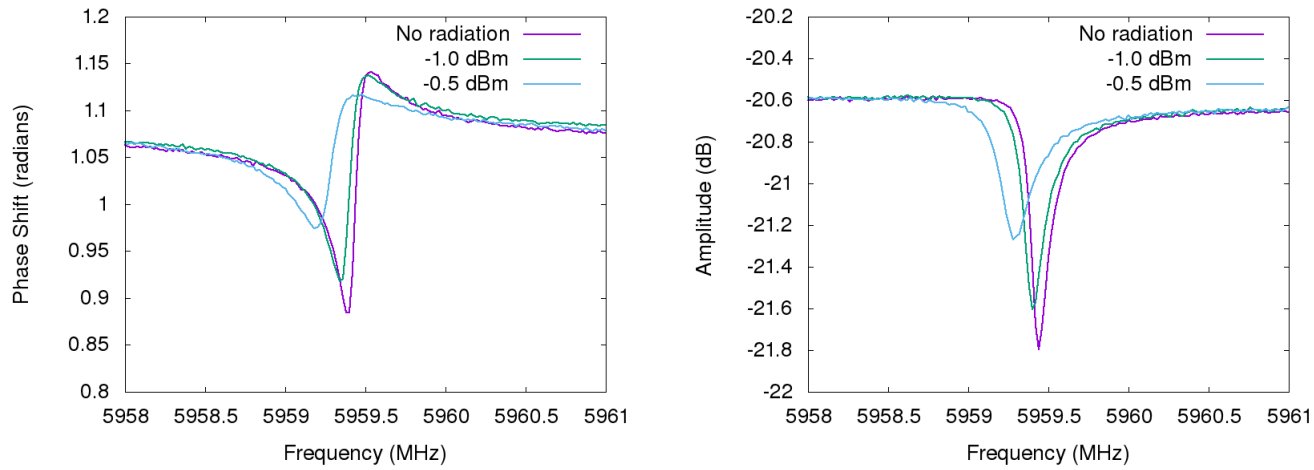

Figure 6. KID response on millimeter wave radiation recorded by our readout system. The left plot shows the phase shift and the right plot shows the amplitude change as a function of the frequency of a KID sensor.

\subsection{Readout Electronics}

For the readout of the KID signal, we use a commercially available field programmable gate array (FPGA) board. At this moment, we use Xilinx Kintex Ultrascale digital board with a daughter board having dual channel ADC and DAC of both 14 bit and 200 MSps. This daughter board, called RHEA board is a homemade board running at $200 \mathrm{MHz}$. A digital radio frequency (RF) waves are generated from FPGA and fed into the DAC. Analog RF signals are modulated with a local oscillator and send to KID sensors. The return signals are demodulated and received by ADC.

Figure 6 show an example of real signal from a KID in a cryostat with the described readout system above. With respect to the resonance curve when there is no radiation, there are clear shifts in phase and in amplitude distribution when millimeter wave radiation at different power levels are applied, which are identical observation with a high quality network analyzer, demonstrating that the basic functionality of the readout system that we developed.

\section{Summary}

The GroundBIRD telescope is aiming at the detection of $B$-mode of CMB polarization down to percent level for $r$. To realize this, a fast rotation of the entire telescope when superconducting millimeter photon sensors are cooling down to $250 \mathrm{mK}$ is implemented. A noble hybrid fabrication method is applied to implement the detector system with both planner and micro strip structure, and its basic principle is demonstrated through comparisons of a prototype with and without antenna circuits. The readout electronics utilize a mixture of commercial FPGA and homemade ADC/DAC boards, and are shown to work as expected. A picture of the assembled GB telescope system is shown in Fig. 7. The current plan is to ship the GB telescope to the observation site in 2018 and commission the GB telescope afterward.

This work is supported by Grants-in-Aid for Scientific Research from The Ministry of Education, Culture, Sports, Science, and Technology, Japan (KAKENHI Grant Nos. 23684017, 25610064, 26105519, 26247045, 14J10972, 15H05448, and 15H05743), the Center for the Promotion of Integrated Sciences (CPIS) of SOKENDAI, the FY 2012 Joint Development Research on an Open Application Basis program of the NAOJ, Research Grants in the Natural Sciences from the Mitsubishi Foundation, and the Basic Science Research Program through the National 


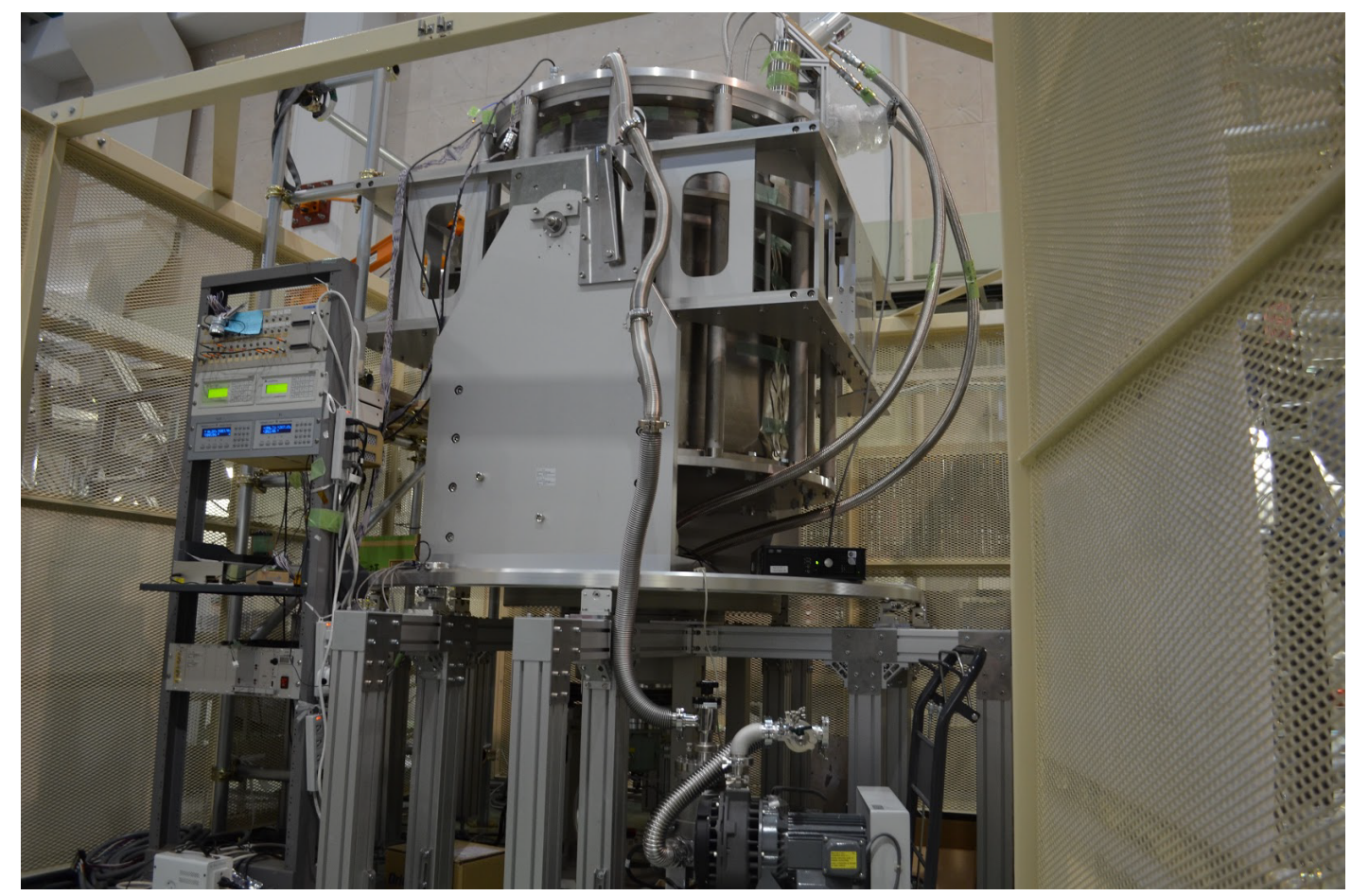

Figure 7. A picture of fully assembled GB telescope in a safety cage. A rack on the left contains service electronics to the telescope.

Research Foundation of Korea (NRF) funded by the Ministry of Education, Science and Technology (Grant No. NRF-2017R1A2B3001968). EW acknowledges partially Korea University Future Research Grant.

\section{References}

[1] S. Dodelson, Modern Cosmology, (Academic Press, Oxford, 2003) 1-20.

[2] The COBE Team: G. F. Smoot et. al, Astrophysical Journal Letters, 396, L1-5 (1992).

[3] W. Rindler, Monthly Notices of the Royal Astronomical Society, 116, 662-677 (1956).

[4] D. N. Spergel, et al., Astrophysical Journal Supplement Series. 170 (2): 337-408 (2007).

[5] R. Dicke, Gravitation and the Universe: Jayne Lectures for 1969, (American Philosophical Society).

[6] A. Guth, Phy. Rev. D 23, 347 (1981); A. Linde, Phys. Lett. 108B, 389 (1982); A. Albrecht and P. J. Steinhardt, Phys. Rev. Lett. 48, 1220 (1982).

[7] U. Seljak, and M. Zaldarriaga, Phys. Rev. Lett. 78, 2054 (1997).

[8] Planck Collaboration, P. A. R. Ade et al., A\&A, 594, A20 (2016).

[9] BICEP2 Collaboration, P. A. R. Ade et al., Phys. Rev. Lett. 116, 031302, (2016).

[10] GroundBIRD Group, S. Oguri et al., J. Low Temp. Phys. 176 691-697 (2014); GroundBIRD

Group, S. Oguri et al., J. Low Temp. Phys. 184 786-792 (2016).

[11] The QUIET Collaboration, Astrophys. J. 741, 111 (2011). 
[12] N. Katayama and E. Komatsu, Astrophys. J. 737, 78 (2011).

[13] Y. Mizuguchi, M. Akagawa, H. Yokoi, in Proceedings of IEEE Antennas and Propagation Society, International Symposium, 14 2-5 (1976).

[14] S. Oguri, J. Choi, M. Kawai, and O. Tajima, Rev. of Sci. Instr. 84, 055116 (2013).

[15] R. Bhatia et al., Cryogenics, 40, 685-691 (2000).

[16] S. Oguri et. al., Rev. of Sci. Instr. 84, 055116 (2013); S. Oguri et. al., Rev. of Sci. Instr. 85, 086101 (2014).

[17] The picture on the right courtesy of K. Kiuchi of RIKEN.

[18] P. Day et. al., Nature, 425, 817 (2003). 